\title{
Hearing Evaluation of Neonates with Hyperbilirubinemia by Otoacoustic Emissions and Brain Stem Evoked Response Audiometry
}

\author{
Mirajkar $\mathbf{S}^{1}$, Rajadhyaksha $\mathbf{S}^{2}$ \\ ${ }^{1}$ Dr. Swapnil Mirajkar, MBBS. MD. ${ }^{2}$ Dr. Surekha \\ Rajadhyaksha, MBBS.MD, DCH. Both from the \\ Department of Paediatrics, BharatiVidyapeeth \\ Deemed University, Pune, Maharashtra, India.
}

\section{Address for correspondence:}

Dr. Swapnil Mirajkar

E-mail: mirajkar.swapnil@gmail.com

\section{How to cite}

Mirajkar S, Rajadhyaksha S. Hearing Evaluation of Neonates with Hyperbilirubinemia by Otoacoustic Emissions and Brain Stem Evoked Response Audiometry. J Nepal Paediatr Soc 2016;36(3):310-313

doi: http://dx.doi.org/10.3126/jnps.v36i3.12155

This work is licensed under a Creative Commons Attribution 3.0 License.

\section{(c) (i)}

\begin{abstract}
Introduction: Neonatal hyperbilirubinemia is one of the most important factors affecting the auditory system and can cause sensorineural hearing loss. This study evaluated the hearing status in neonates with hyperbilirubinemia and results obtained from Otoacoustic emissions (OAE) and Brain stem evoked response audiometry (BERA) analysis were compared with each other's. Material and Methods:This study was performed on fifty eligible term neonates with hyperbilirubinemia requiring either phototherapy or exchange transfusion or both. Hearing analysis was done by OAE and BERA. Results: Out of fifty eligible neonates, twenty one (42\%) had hearing impairment as per OAE analysis, eight (16\%) had hearing impairment; two $(4 \%)$ neonate had inconclusive hearing analysis as per BERA analysis. Out of twenty one neonates having hearing impairment by OAE only five had hearing impairment as per BERA analysis. While the eight neonates who had hearing impairment by BERA analysis, five had hearing impairment by OAE. Conclusion: The study showed hearing impairment by OAE analysis in $42 \%$ neonates while the BERA analysis showed hearing impairment in $16 \%$ neonates. The increased level of Serum bilirubin has not shown the increase in percentage of hearing impairment.
\end{abstract}

Key words: Neonates, hyperbilirubinemia, hearing evaluation, OAE, BERA

\section{Introduction}

T he elevation of serum bilirubin levels in the normal newborn is caused by the greater concentration of circulating erythrocytes and the infant's diminished ability to conjugate and excrete bilirubin because of a lack of the enzyme glucuronyltransferase, a reduced albumin concentration, and a lack of intestinal bacteria ${ }^{1}$.

Acute bilirubin encephalopathy may develop during hazardous hyperbilirubinemia and evolve into the chronic adverse neurodevelopmental sequelae of kernicterus, which is devastating, disabling neurologic disorder classically characterized by the clinical tetrad of 1) ChoreoathetoidCerebral Palsy, 2) High frequency central neural hearing loss, 3) Palsy of vertical gaze, and 4) Dental enamel hypoplasia, the result of bilirubin induced cell toxicity ${ }^{2}$. The CNS 
sequelae reflect both a predilection of bilirubin toxicity for neurons (rather than glial cells) and the regional topography of bilirubin induced neuronal injury that is characterized by the prominent basal ganglia, cochlear, and oculomotar nuclei involvement.

The ability to hear during early months of life is important for the development of speech, language and cognition. Late detection of hearing loss causes irreversible stunting of the language development potential of the child.

Facilities available for detection of hearing loss in the neonates are Otoacoustic Emissions (OAE) and Automated brain stem responses (ABR) or Brainstem Evoked Response Audiometry (BERA). The OAE test evaluates the function of peripheral auditory system, primarily the cochlea which is most often involved in sensorineural hearing loss ${ }^{3}$.Simplicity of the test and fair reliability of results has made OAE test popular in recent years.

Auditory brainstem response (ABR) typically uses a click stimulus that generates a response from the basilar region of the cochlea. The signal travels along the auditory pathway from the cochlear nuclear complex to the inferior colliculus ${ }^{4}$.

The associated hearing defects due to elevated levels of neonatal jaundice are the reason for interest on this subject.

\section{Materials and Methods}

The study was performed from October 2012 to September 2014 at tertiary care centre in Pune. During the study period there were $2704 \mathrm{NICU}$ admissions out of which 575 neonates had hyperbilirubinemia requiring treatment in form of phototherapy and or exchange transfusion as per criteria laid by American Academy of Paediatrics $2004^{5}$. Out of them the neonates who fulfilled the inclusion criteria and whose parents were ready for further evaluation by OAE and BERA were 50 . Inclusion criteria was full term (more than 37 weeks of the gestational age) neonates with hyperbilirubinemia (as per criteria laid by American academy of Paediatrics 2004). Neonates with prematurity (less than or equal to 37 weeks), history of birth asphyxia, craniofacial anomalies, familial history of hearing loss, Infections (TORCH), Sepsis, Meningitis were excluded.

Hypebilirubinemic neonates within inclusion criteria underwent Oto-Ocustic Emissions (OAE) just prior to discharge from NICU or on day of discharge. Brainstem Evoked Response Audiometry (BERA) was done as per appointment given by audiology department (shortly after discharge) on same babies. The working definitions for hyperbilirubinemia, need for phototherapy and exchange transfusion was taken as per guidelines laid by American academy Paediatrics 2004.

\section{Results}

During the study period there were 2704 NICU admissions out of which 575 neonates had hyperbilirubinemia requiring treatment in form of phototherapy and or exchange transfusion. Out of them the neonates who fulfilled the inclusion criteria and whose parents were ready for further evaluation by OAE and BERA were fifty.

In our study the lowest Serum bilirubin was $12 \mathrm{mg} / \mathrm{dl}$ in which Rh incompatibility was the cause of hyperbilirubinemia of the same neonate whose Serum bilirubin was $12 \mathrm{mg} / \mathrm{dl}$. There was no hearing impairment in the same neonate. While the highest Serum bilirubin was $39.7 \mathrm{mg} / \mathrm{dl}$. OB blood group set up (mother's blood group $\mathrm{O}$ and neonate's blood group $\mathrm{B}$ i.e. OB incompatibility) was the cause of hyperbilirubinemia here, but the hearing evaluation was also normal in this neonate.

The OAE analysis in fifteen (30\%) neonates out of fifty showed as bilaterally REFER meaning failed, while in two (4\%) neonates it showed right REFER and four (8\%) neonates had left REFER.

Twenty nine (58\%) neonates had their OAE analysis as PASS meaning that it is normal.

Of the fifty neonates, the BERA analysis of three $(6 \%)$ neonates had left mild hearing loss, three $(6 \%)$ neonates had right sided mild hearing loss, one $(2 \%)$ neonate had bilateral moderate to moderately severe hearing loss, one $(2 \%)$ neonate had bilateral mild hearing loss, and two (4\%) neonates had their BERA analysis as inconclusive. Forty $(80 \%)$ neonates had their BERA analysis as normal.

Correlation of OAE and BERA: Out of fifteen neonates who's OAE showed bilateral REFER, three had left mild hearing loss and one had right mild hearing loss according to BERA analysis. Rest eleven neonates had normal BERA analysis.

Correlation of BERA and OAE: The BERA analysis which showed bilateral hearing loss in two neonates, had normal OAE in both those neonates. Out of three neonates which showed right hearing loss by BERA, two neonates OAE showed REFER while one had 
Table 1: Distribution of neonates according to severity of hyperbilirubinemia and their OAE and BERA analysis.

\begin{tabular}{ccccccc}
\hline $\begin{array}{c}\text { Serum Bilirubin } \\
\mathbf{m g} / \mathbf{d l}\end{array}$ & $\begin{array}{c}\text { Total } \\
\text { neonates }\end{array}$ & $\begin{array}{c}\text { Abnormal } \\
\text { OAE }\end{array}$ & $\begin{array}{c}\text { Abnormal } \\
\text { BERA }\end{array}$ & $\begin{array}{c}\text { Both OAE and } \\
\text { BERA Abnormal }\end{array}$ & $\begin{array}{c}\text { Inconclusive } \\
\text { Hearing }\end{array}$ & $\begin{array}{c}\text { Normal } \\
\text { Hearing }\end{array}$ \\
\hline $11-15$ & 6 & 1 & 1 & 0 & 1 & 3 \\
$>15-20$ & 23 & 6 & 1 & 3 & 0 & 13 \\
$>20-25$ & 9 & 4 & 1 & 2 & 0 & 2 \\
\hline$>25$ & 12 & 5 & 0 & 0 & 1 & 6 \\
\hline
\end{tabular}

PASS of same ear. While the three neonates who had left hearing loss by BERA, all these three neonates had REFER OAE of same ear.

\section{Discussion}

In our study it is seen that even in the neonates with lower bilirubin, i.e. of range of $11 \mathrm{mg} / \mathrm{dl}$ to $15 \mathrm{mg} / \mathrm{dl}$ there was hearing impairment in two. One had abnormal OAE and one had abnormal BERA. In neonates with higher Serum bilirubin i.e; in range of serum Bilirubin more than $25 \mathrm{mg} / \mathrm{dl}$, five neonates had abnormal OAE but the BERA analysis of same neonates showed no hearing abnormality.

The percentage of hearing impaired neonates is not increasing with the increase in serum Bilirubin. In the study of Jiang et al in 2007 on 90 infants with a history of neonatal hyperbilirubinemia, it was described that recorded thresholds and response latencies of all waves showed considerable increases and the same time, there was no remarkable correlation between serum bilirubin concentration and increased latencies ${ }^{6}$. Moreover, Jiang and colleagues in 2009 in a study on eighty-three term infants with serum bilirubin levels more than $10 \mathrm{mg} / \mathrm{dL}$ exhibited a significant decrease in the amplitude of waves III and V. However, none of the amplitude values obtained had a considerable relation with total serum bilirubin levels.

Overall the OAE analysis showed hearing impairment in $42 \%$ neonates. Baradaranfar $\mathrm{MH}$, Atighechi $\mathrm{S}$ et al performed OAE on thirty-five neonates with hyperbilirubinemia, thirty cases $(85.7 \%)$ passed whereas the remaining (14.3\%) seemed to be failures ${ }^{7}$. The result of our study showed much high hearing impairment because of high false positivity of OAE.
The BERA analysis was abnormal in $16 \%$ neonates while $84 \%$ neonates had normal hearing analysis. Baradaranfar $\mathrm{MH}$, AtighechiSet all performed BERA on thirty-five neonates with hyperbilirubinemia. BERA results indicated that twenty-six cases $(74.3 \%)$ had normal hearing but nine $(25.7 \%)$ suffered from an impairment $^{7}$. Our result is in consistence with the study done.

In our study, out of twenty-one neonates whose OAE reported as REFER, only five neonates had hearing impairment by BERA. Silva DP, Martins RH used transient evoked otoacoustic emissions and brainstem evoked auditory potentials and concluded that in neonates with hyperbilirubinemia, have lower transient evoked otoacoustic emissions amplitudes and mild PV and LI-V increase, indicating cochlear and retrocochlear disorders, stressing the importance of using both tests ${ }^{8}$.

In our study out of eight neonates whose BERA was abnormal only five neonates had failed in OAE. Granell J, Gavilanes et all compared a group of 862 newborns from year 2003, screened with transient evoked OAE with a clinical device, with a group of 2300 newborns from years 2005 and 2006, screened with automated BERA and found a statistically significant difference in the percentage of pass in the first step, favouring BERA $(99.7 \% \text { vs } 91.8 \% ; p<.0005)^{9}$.

\section{Conclusion}

The study showed hearing impairment by OAE analysis in $42 \%$ neonates while the BERA analysis showed hearing impairment in $16 \%$ neonates. The increased level of serum bilirubin did not show increase in percentage of hearing impairment. The hearing impairment should be confirmed by repeat BERA after three months of age. 


\section{References}

1. Dennery, PA, Seidman, DS, and Stevenson, DK. Neonatal hyperbilirubinemia. NEJM 2001;344:581-90.

2. Shapiro, S. M. \& Nakamura, H. (2001). Bilirubin and the auditory system. JPerinatol 2001;21:S52-S55.

3. Vohr BR, et all. Identification of neonatal hearing impairment: characteristics ofinfants in the neonatal intensive care unit and well-baby nursery. Ear \& Hearing; 200;21(5):373-382

4. Sininger, Y. \& Cone-Wesson, B. (2002). Threshold prediction using auditory brainstem response and steady-state evoked potentials with infants and young children. In J. Katz (Ed.): Handbook of Clinical Audiology. 5th ed. Baltimore, Maryland: Lippincott, Williams \& Wilkins; 298-322.

5. American Academy of Pediatrics Clinical Practice Guideline Subcommittee on Hyperbilirubinemia
Management of Hyperbilirubinemia in the Newborn Infant 35 or More Weeks of Gestation. Pediatrics 2004;114(1)297-316.

6. Jiang ZD, Chen C, Liu TT, Wilkinson AR. Changes in brainstem auditory evoked response latencies in term neonates with hyperbilirubinemia. PedtrNeurol 2007;37(1):35-41.

7. Baradaranfar $\mathrm{MH}$, AtighechiSet all, Hearing status in neonatal hyperbilirubinemia by auditory brain stem evoked response and transient evoked otoacoustic emission. Acta Med Iran 2011;49(2):109-12.

8. Silva DP, MartinsRH. Analysis of transient otoacoustic emissions and brainstem evoked auditory potentials in neonates with hyperbilirubinemia. Braz J Otorhinolaryngo/2009;75(3):381-6.

9. Granell J, Gavilanes J et al, Is universal newborn hearing screening more efficient with auditory evoked potentials compared to otoacoustic emissions. ActaOtorrinolaringolEsp2008;59(4):170-75. 